\section{Major psychiatric disorders increase risk of mortality}

\section{QUESTION}

Question: How do death rates differ across cohorts with major psychiatric disorders?

Population: 5558959 individuals born in Denmark who were alive on or born after 1 January 1973 and were still alive at their 15th birthday were included; information taken from the Civil Registration System.

Setting: General population, Denmark; registry data examined from 1 January 1973 to 1 January 2001.

Prognostic factors: Schizophrenia, schizoaffective disorder, unipolar depressive disorder, or bipolar affective disorder. Data on admissions to psychiatric inpatient services and diagnoses were available through the Danish Psychiatric Central Register. Outcomes: Mortality rate, cause of death (from the Civil Registration System and the Cause of Death register).

\section{METHODS}

Design: Retrospective cohort study.

Follow-up period: From age 15 years or on 1 January 1973 (whichever came later) to date of death, date of emigration or 1 January 2001 (whichever came first).

\section{MAIN RESULTS}

Mortality rates differed across the cohort depending on psychiatric diagnosis. All people admitted for any major psychiatric disorder were more likely to die from natural or unnatural causes than people who were not admitted (mortality rate ratio: $9.44,95 \%$ CI 9.12 to 9.78). When admissions were broken down by diagnosis, people with schizoaffective disorder were at the highest risk of death compared with non-admitted people (mortality rate ratio: $12.58,95 \%$ CI 9.96 to 15.89 ). When deaths were broken down into natural and unnatural causes, unnatural deaths contributed most to the risk of death (mortality rate ratio for unnatural death with any admission: $14.52,95 \%$ CI 13.85 to 15.23). Suicide contributed most to the elevated risk of unnatural death with any admission (suicide rate ratio for males with any admission: 17.26, 95\% CI 16.72 to 17.82; suicide rate ratio for females with any admission: $29.08,95 \%$ CI 27.89 to 30.31). Other causes of death examined included cardiovascular disease, respiratory diseases, cancer, homicide, endocrine and metabolic conditions, old age and accidents. An increased mortality rate ratio for all of these causes was seen in all diagnostic groups, except for cancer in schizoaffective disorder and bipolar disorder.

\section{CONCLUSIONS}

Persons admitted with one of four major psychiatric disorders (unipolar disorder, bipolar disorder, schizoaffective disorder or schizophrenia) are at greater risk of mortality, particularly from unnatural causes (including suicide), than people who are not admitted.

\section{ABSTRACTED FROM}

Laursen TM, Munk-Olsen T, Nordentoft M, et al. Increased mortality among patients admitted with major psychiatric disorders: a register-based study comparing mortality in unipolar depressive disorder, bipolar affective disorder, schizoaffective disorder, and schizophrenia. J Clin Psychiatry 2007; 68:899-907.

Notes: Overlaps in the diagnostic groups (for example, where an individual had more than one diagnosis) were not censored out of analyses. In analyses, people admitted with more than one diagnosis were included in all diagnostic groups into which they were categorised.

Correspondence to: Thomas Munk Laursen, MSc, PhD, National Centre for RegisterBased Research, University of Aarhus, Taasingegade 1, DK-8000 Aarhus C, Denmark; tml@ncrr.dk

Source of funding: The Stanley Medical Research Institute, Chevy Chase, MD and the Center for Basic Psychiatric Research, Aarhus, Denmark.
E pidemiological studies have consistently found excess mortality rates among individuals with diagnoses of serious mental illness (schizophrenia, schizoaffective disorder and bipolar and unipolar affective disorders) compared with general population rates. ${ }^{1}$ Laursen and colleagues replicate these findings in a register-based study that permitted a unique comparison of the magnitude of excess mortality across diagnostic, age and gender groups by cause of death. All four diagnostic groups experienced remarkably similar degrees of excess mortality, a finding relatively new to the literature. Another unique finding is the increase in excess mortality contributed by the presence of one or more first degree relative with a psychiatric admission, possibly suggesting shared genetic or environmental influences on mortality.

The reported similarity of overall mortality, in addition to the results of a recent extensive systematic review of mortality in schizophrenia, ${ }^{2}$ supports the likelihood that these findings are reasonably generalisable to other settings.
The results of this study have important implications for clinical practice. The greatest excess mortality risk was found for unnatural causes of death, chiefly suicide, a not unexpected finding which emphasises the need for aggressive suicide prevention strategies. However, in terms of absolute mortality, cardiovascular and other natural causes of death make by far the greatest contribution to premature death among the seriously mentally ill. ${ }^{3} \mathrm{~A}$ recent commentary focused on cardiovascular disease ${ }^{4}$ underscored both the excess prevalence of risk factors for these disorders among individuals with severe mental illness (for example, comparatively high prevalence of smoking, diabetes, dyslipidaemia, hypertension or obesity) and the relative lack of effective treatment for these conditions in US academic and public sector mental health treatment settings. These findings plus recent evidence that the mortality gap for schizophrenia may be worsening over time, ${ }^{2}$ together with the increased liability of some antipsychotic agents to adversely affect adiposity and glucose and lipid metabolism, ${ }^{4}$ clearly point to the need for changes in practice to ensure the recognition, prevention and treatment of these modifiable risk factors. In particular, there is a need for closer collaboration between mental health and general medicine practitioners plus access to specific treatment programmes (for example, smoking cessation) if the observed mortality gap is to be reduced.

\section{Thomas J Craig, MD, MPH}

Springfield, Virginia, USA

\section{Competing interests: None.}

1. Harris EC, Barraclough B. Excess mortality of mental disorder. Br J Psychiatry 1998;173:11-53.

2. Saha S, Chant D, McGrath J. A systematic review of mortality in schizophrenia: is the differential mortality gap worsening over time? Arch Gen Psychiatry 2007; 64:1123-31.

3. Colton CW, Mandersheid RW. Congruencies in increased mortality rates, years of potential life lost, and causes of death among public mental health clients in eight states. Prev Chronic Dis 2006;3:A42.

4. Newcomer JW, Hennekens CH. Severe mental illness and risk of cardiovascular disease. JAMA 2007;298: 1794-6. 